Ecología / Ecology

\title{
USO DE HERRAMIENTAS DE SISTEMAS DE INFORMACIÓN GEOGRÁFICA PARA ESTABLECER LA ZONIFICACIÓN ECOLÓGICA DE UNIDADES DE PAISAJE EN UN SECTOR DEL MUNICIPIO DE TIMBÍO - CAUCA
}

\section{Use of geographical information systems tools to establish the ecological zoning of landscape units in a sector of the municipality of Timbío - Cauca}

\author{
Julieth Alexandra Chacón Paja ${ }^{1}$ \\ Carolina Bambagüé Caicedo ${ }^{2}$ \\ Older Enrique Arboleda Riascos
}

1 Ingeniera Forestal, grupo Unidad de Investigación en Ecología Tropical UNIET, Programa de Ecología, Fundación Universitaria de Popayán. Dirección: Calle $73 A N 12^{a} 63$ CASA D6. Villas de San Sebastián. Popayán, Cauca. E-mail: julieth.chacon@docente.fup.edu.co

2 Ecóloga, Programa de Ecología, Fundación Universitaria de Popayán. E-mail: carobc14@gmail.com

3 Ecólogo, Programa de Ecología, Fundación Universitaria de Popayán. E-mail:oldero4@hotmail.com

\section{Resumen}

La zonificación ecológica considera el paisaje como una entidad espacio temporal integrada, contribuyendo a presentar la dinámica de los procesos ecológicos y funcionamiento del mismo haciendo uso de los Sistemas de Información Geográfica (SIG) ya que permiten conocer, planear y ordenar el territorio. Este
Historia del artículo

Fecha de recepción: 10/06/2020

Fecha de aceptación: $16 / 07 / 2020$ 
estudio se realizó en el Jardín Botánico de Popayán (JBP) y su área de influencia, donde se desarrollan procesos de investigación y educación ambiental que hacen parte de la zona de conservación perteneciente a la sede campestre de la Fundación Universitaria de Popayán (FUP), en la vereda Los Robles, como un modelo a pequeña escala para la zonificación ecológica de las unidades de paisaje del municipio de Timbío (Cauca). Por lo anterior, se hizo uso de los SIG y revisión de información secundaria del área de estudio para la obtención de siete mapas finales que evidencian las unidades de paisaje, coberturas vegetales, uso actual de suelo, aptitud de suelo, conflicto de uso de suelo y finalmente la espacialización de la zonificación ecológica. Para ello se tuvieron en cuenta las siguientes zonas (Z): Z de especial significado ambiental (31,6 ha); Z de recuperación ambiental (4,4 ha); Z de riesgo y amenazas (o,2 ha); $\mathrm{Z}$ de producción económica (7,7 ha) y Z de importancia social (3,6 ha). Permitiendo establecer estrategias de conservación para elJBP, bajo tres líneas operativas, cada una con un proyecto a emprender: 1. Línea de investigación: La biodiversidad como estrategia de conservación en el JBP; 2. Línea de educación ambiental: La educación ambiental como herramienta pedagógica para la conservación de la biodiversidad; 3. Línea de uso sostenible de la biodiversidad: El desarrollo sostenible un tema importante en la conservación de biodiversidad.

Palabras clave: Ecología del paisaje; zonificación ecológica; coberturas vegetales; conservación; Sistema de Información Geográfico SIG

\section{Abstract}

Ecological zoning considers the landscape as an integrated temporal space entity, helping to present the dynamics of ecological processes and the operation of the same by making use of geographic information systems (G.I.S) since it allows knowing, planning and ordering the territory. This study was carried out in the Botanical Garden of Popayan (J.B.P) and its area of influence where research processes and environmental education are carried out, which are part of the conservation area belonging to the country headquarters of the Popayán University Foundation, in the los Robles Village in order to be a small-scale model for the ecological zoning of landscape units in the Municipality of Timbío. Therefore, GIS was used and secondary information was reviewed in the study area to obtain seven final maps that show the landscape units, vegetation cover, current land use, land suitability, land use conflict, and finally, the spatialization of ecological zoning where we took into account the following areas (Z): Z. of special environmental significance (31.6 ha); Z. of environmental recovery (4.4 ha); Z. of risk and threats (o.2 ha); Z. of economic production (7.7 ha) and Z. of social importance (3.6 ha). Allowing to establish conservation strategies for the JBP, under 3 operational lines each with a project to undertake: 1 . Research line: Biodiversity as a conservation strategy in the JBP. 2. Environmental education line: Environmental education as a pedagogical tool for the conservation of biodiversity. 3. Sustainable use of biodiversity line: 
Sustainable development is an important issue in the conservation of biodiversity.

Keywords: Landscape ecology; ecological zoning; vegetation cover; conservation; Geographic Information System (SIG).

\section{Introducción}

La zonificación ecológica es la base para determinar cómo se debe utilizar apropiadamente el territorio con criterios de equidad y cohesión territorial en relación con el equilibrio ambiental, por lo cual se comprende como "el conjunto de elementos bióticos y abióticos que dan sustento a los procesos ecológicos esenciales del territorio, cuya finalidad principal es la preservación, conservación, restauración, uso y manejo sostenible de los recursos naturales renovables, para el desarrollo socioeconómico de las poblaciones (Villegas et al., 2015). Así mismo, la zonificación ecológica aplica fundamentos del enfoque estructural de la ecología del paisaje, al integrar de manera jerárquica características espaciales y estructurales de elementos biofísicos como el clima, geología, geomorfología, suelos y coberturas que se integran conformando unidades homogéneas de territorio con características biofísicas comunes (Serrato, 2018).

En la última década se han venido realizando estudios a nivel de Latinoamérica sobre zonificación ecológica usando los Sistemas de Información Geográfico SIG, que se han convertido en una herramienta para la planificación, cuyo uso se ha extendido por el tipo de análisis que genera y que ha permitido la incorporación de diferentes variables para efectuar una interpretación geográfica adecuada para la planeación y organización del territorio (Marín y Salazar, 2020). Para este estudio se hizo uso de un vehículo aéreo no tripulado (dron) permitiendo obtener un producto fotogramétrico de alta resolución espacial, disminuyendo tiempos y costos en levantamientos topográficos, generando un producto geoespacial para la delimitación y caracterización de las unidades de paisaje con el fin de realizar la zonificación ecológica, siendo este un insumo base para la elaboración del Plan de Manejo Ambiental (PMA) delJBP, además de ser un modelo para la zonificación ecológica del Municipio de Timbío, en el cual se encuentran relictos de bosque subandino con características de vegetación de bosque secundario sucesional, bajo diferentes grados de intervención de actividades antrópicas evidenciadas por zonas de expansión agropecuaria y fragimentación de bosque.

\section{Materiales y métodos}

\section{Área de estudio}

El trabajo de campo se realizó en el Campus Los Robles de la Fundación Universitaria de Popayán, ubicado en la vereda Los Robles, Municipio de Timbío, a 8km del sur occidente de Popayán, departamento del Cauca y registra las siguientes coordenadas geográficas: $\mathrm{N} \mathrm{O2}^{\circ} 23^{\circ} 28.7^{\prime \prime} \mathrm{W} \mathrm{o} 76^{\circ} 399^{\prime} 24.9$ " con altitud 1850 msnm (Mapa 1). Según el sistema de clasificación de zonas de vidas de Holdridge, se clasifica en bosque subandino, 


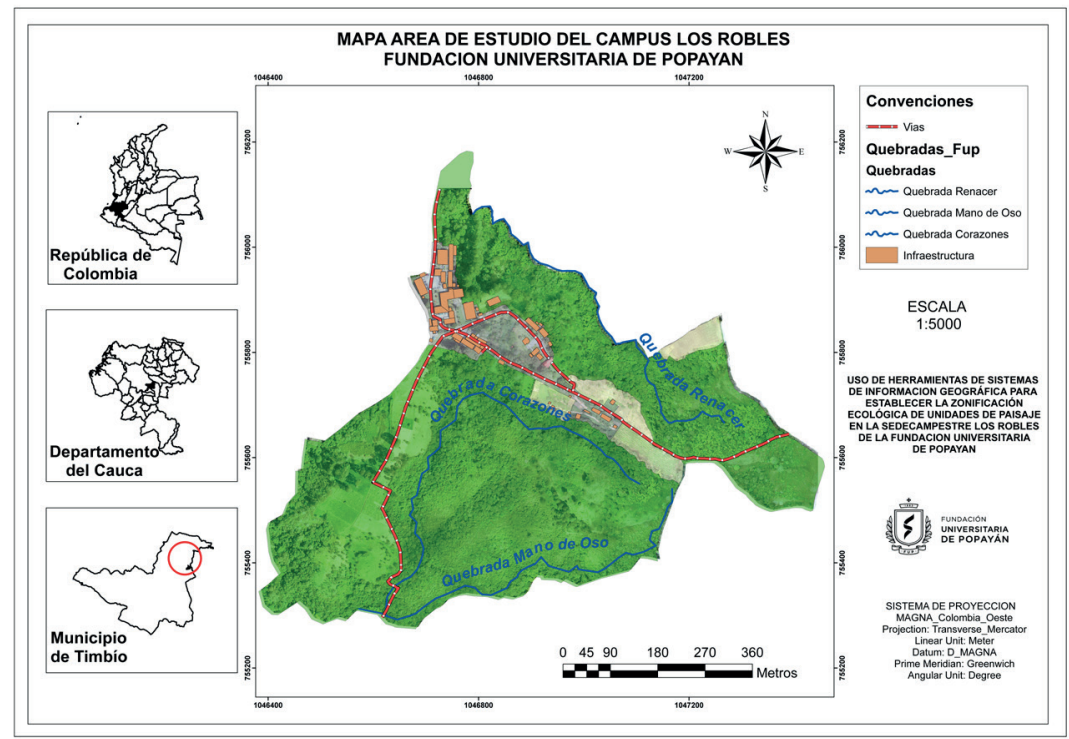

Mapa 1. Localización del área de estudio.

siendo la vegetación principal del área un relicto de bosque subandino secundario. Cuenta con un área boscosa en estado de sucesión secundaria y es considerado zona de conservación de especies nativas, con la finalidad de desarrollar procesos de investigación, conservación, exhibición y educación además esta área de estudio funciona como corredor biológico para conexión de los diferentes parches boscosos contribuyendo a la conservación de la biodiversidad.

En cuanto aspectos abióticos de la zona de estudio se mencionan los siguientes:

a. Clima: corresponde a tropical lluvioso, se caracteriza por presentar condiciones de tipo bimodal. Méndez y Vallejo (2003) mencionan que las épocas de lluvias fuertes son en marzo - mayo, lluvias menos fuertes en octubre - diciembre y épocas secas en enero - febrero, junio septiembre. Según López y Bastidas (2010), la precipitación anual promedio es de 2200omm. Temperatura media de $18.7^{\circ} \mathrm{C}$; humedad relativa entre $63 \%-81 \%$. Las nubes más frecuentes son cúmulos, altocúmulos, estratocúmulos, stratus, stratusfractus y cirrostratos, permitiendo 1350 horas/año de insolación promedio (Negret et al., 1997).

b. Hidrología: El Jardín Botánico de Popayán tiene dos quebradas principales, Renacer y Mano de Oso, además de las quebradas corazones y Wettu; los drenajes están controlados por los ríos Robles, Timbío, Piedras y las vertientes San Jorge, El muerto, El hato Nuevo y Cenaqueras (Güetio y Solarte, 2000). La quebrada renacer se encuentra ubicada al Noreste del Jardín Botánico con una longitud de 778 metros (Bambagüé y 
Arboleda, 2017), bordea un bosque que se caracteriza por ser denso, con menor grado de conservación, empleado para actividades de guianza interpretativa del JBP, con transición de cultivos de café en su gran mayoría y zonas inundables de alta importancia para grupos de anfibios y reptiles. Cuenta con 37 especies de plantas leñosas, principalmente representadas por Palicourea thyrsiflora (Cafetillo) y Alchornea coelophylla (Lombricero) (Chaves y Hurtado, 2019).

c. Geología y geomorfología: De acuerdo al estudio general de suelos para el departamento del Cauca, esta área se ubica en lomas y colinas en el paisaje de lomerío, el relieve es ligeramente ondulado a moderadamente escarpado con pendientes hasta del $75 \%$ rectas, convexas y medias. Los suelos se han derivados de capas de cenizas volcánicas discontinuas sobre rocas ígneas mixtas (andesitas, tobas, y brechas). Son bien drenados, profundos, texturas moderadamente gruesas, muy fuerte, ligeramente ácidos, alta saturación de aluminio, algunos suelos presentan erosión hídrica moderada o severa causadas por las aguas de escorrentías que forman cárcavas, están conformados por suelos Dystrudepts (35\%), Hapludoxs (30\%), Hapludands (25\%) y Udorthents (10\%) (IGAG, 2009).

Los parámetros básicos de información del territorio y la identificación previa de las unidades de paisaje existentes, permitieron la estructuración y ruta metodológica para el desarrollo de la zonificación ambiental. Se describe la ruta metodológica que se dividió en cuatro fases como se muestra a continuación:

\section{Fase I. Reconocimiento zona de estudio.}

Se realizó la consulta bibliográfica de aspectos ambientales de la zona de estudio, como insumo para el análisis de los mapas obtenidos y para formular las estrategias de conservación en elJBP. Seguidamente se procedió a la fotointerpretación preliminar de imágenes provenientes de visores geográficos como Google Earth y Arcgis online, además se consultaron planchas cartográficas en formato digital y análogo del instituto Geográfico Agustín Codazzi del año 2016; en esta actividad se reconoció la zona de estudio, se registraron en GPS Garmin ETrex 30 las coordenadas geográficas y puntos de muestreo de interés, como las coberturas vegetales y microcuencas; información que permitió la delimitación del área de estudio e identificación preliminar de posibles unidades de paisaje.

Fase II. Levantamiento y procesamiento de información espacial.

Haciendo uso y vuelo del dron 3D Robotics Iris se tomaron coordenadas de la zona, seguidamente se dividió el trabajo en cuatro misiones, con el objetivo de captar el área de estudio. El primer vuelo capturó 75 imágenes, el segundo vuelo 74; tercer vuelo 77 y finalmente el cuarto vuelo 72 , para un total de 298 fotografías que conformaron la ortoimagen de referencia del área de estudio (Figura 1). Para el procesamiento de las imágenes tomadas con el dron se utilizó el 


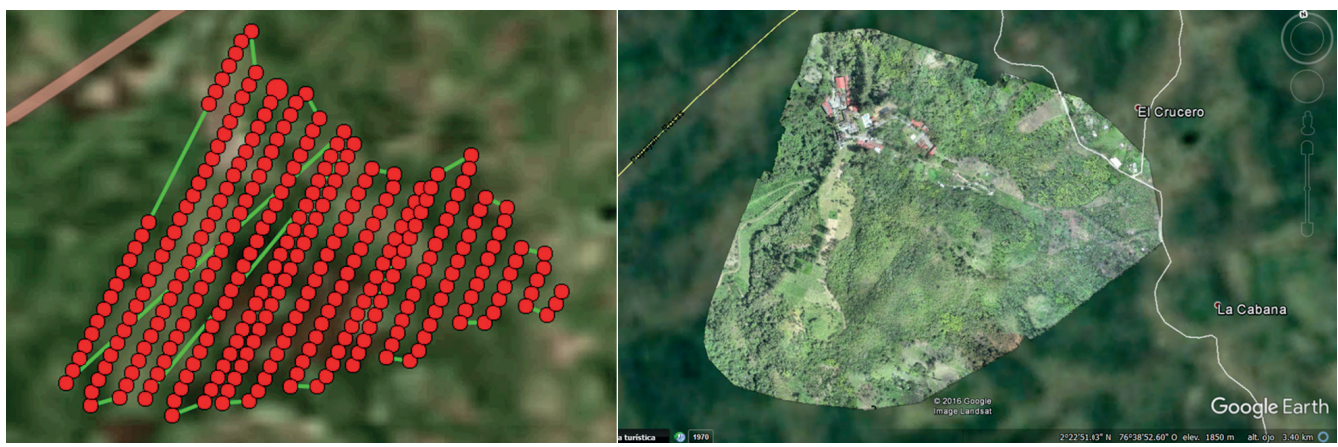

Figura 1. Líneas de Vuelo y generación de ortoimagen con Drone 3D Robotics Iris.

programa Pix4DMapper Versión Profesional - 2017. Se llevó a cabo la generación de la nube de puntos, conocido como modelo digital de superficie y ortoimagen.

Una vez se obtuvo la ortoimagen de la zona de estudio, se procedió a registrar la información de y los puntos tomados con el GPS en ArcMap, aplicación principal de ArcGIS versión 10.3. En seguida se hizo la interpretación preliminar de la imagen, identificando las zonas recorridas en campo como coberturas vegetales, cómo se encuentra distribuido el territorio y otras áreas de interés. Posteriormente se utilizó la herramienta de ArcMap "Analysis tools" para realizar la edición vectorial, creando capas a partir de la fotointerpretación de la ortoimagen, además del uso de herramientas de geoprocesamiento como unión, clip e intersect, que permiten llevar a cabo diferentes análisis y extraer nuevos valores a partir de los contenidos en una o varias capas. Así se obtuvo el mapa general de la zona de estudio, mapa de unidades de paisaje, mapa de coberturas vegetales, mapa de uso actual, mapa aptitud del suelo, mapa de conflicto de uso y finalmente el mapa de zonificación ecológica. Además, se creó e implementó la base de datos que contiene el nombre de las coberturas y unidades de paisaje de las capas que se construyeron, además de la medición de cada una de las áreas para, más adelante, realizar cambios o adicionar información relevante, que finalmente contribuyeron a la generación de la cartografía digital.

Para la clasificación de unidades de paisaje se utilizó la metodología del Estudio general de suelos y zonificación de tierras departamento del Cauca a Escala 1:100.000 (2009). Por otro lado, para la clasificación de coberturas se utilizó la Leyenda nacional de coberturas de la tierra, Metodología CORINE Land Cover adaptada para Colombia a Escala 1:100.000 (2010). Este sistema de clasificación consiste en una nomenclatura de tipo jerárquica, que consta de categorías con información a nivel regional, las cuales pueden ser agregadas en niveles superiores de las categorías a nivel nacional, lo cual facilita el manejo de información para la ordenación del territorio. Para este estudio se llegó hasta la clasificación 3 como nivel de detalle (IGAC, 2007). 
Fase III. Elaboración de la cartografía.

Para esta fase, se utilizó el software ArcGIS versión 10.3 haciendo uso de la aplicación Arcmap. Las herramientas que se utilizaron fueron: manejo de vistas, simbología y etiquetas, geoprocesamiento, layouts o planchas, proyecciones, georreferenciación y digitalización. Se procedió a la fotointerpretación de la ortoimagen con su respectiva verificación en campo para la elaboración de las distintas capas usadas para la generación de mapas, superposición de mapas iniciales y posteriormente el análisis de resultados de los mapas intermedios, continuando con la superposición de estos y el análisis de la situación actual para generar el mapa de conflicto de uso. Finalmente se elaboró la zonificación ecológica, a partir de la evaluación del área de estudio, teniendo en cuenta la información obtenida de los mapas; luego se determinaron las zonas definidas en la guía del Ministerio de Ambiente y Desarrollo Sostenible "Metodología general para la elaboración de estudios ambientales" (2010).

Fase IV. Formulación de estrategias de conservación.

A partir de la información obtenida en las etapas anteriores, se elaboró una matriz DOFA, en la cual se identificaron oportunidades, debilidades, amenazas y fortalezas, para la formulación de estrategias de conservación para elJBP, que está dentro de la zona de estudio, asimismo, se tuvo en cuenta el Plan nacional de colecciones de Jardines Botánicos en Colombia,

\section{Resultados y discusión}

Caracterización de las unidades de paisaje.

Para caracterizar estas unidades se tomaron en cuenta aspectos abióticos, los cuales fueron mencionados en descripción del área de estudio, y aspectos bióticos, los cuales se especifican a continuación:

\section{Aspectos bióticos}

a. Formaciones vegetales: Según la clasificación de Cuatrecasas (1958) al área de estudio pertenece a la formación vegetal de selva Neotropical - Subandina que va desde de los 1000 hasta 2400 metros y precipitaciones regularmente distribuidas de 1000 a $4000 \mathrm{~mm}$ anuales, las temperaturas medias anuales están entre $\operatorname{los} 23^{\circ}$ y $16^{\circ} \mathrm{C}$. La selva Subandina se divide en dos pisos altitudinales diferentes, uno a los $1400 \mathrm{~m}$ y el otro a los $2000 \mathrm{~m}$, para los $1400 \mathrm{msnm}$ se encuentran árboles de 15 a 30 m de altura de las familias Apocynaceae, Annonaceae, Araliaceae, Brunelliaceae, Burseraceae, Clusiaceae, Elaeocarpaceae, Euphorbiaceae, Flacourtiaceae, Hypericaceae, Icacinaceae, Lauraceae, Lecythidaceae, Leguminosae, Melastomataceae, Meliaceae, Moraceae, Myristicaceae, Proteaceae, Rubiaceae, Sapindaceae, Solanaceae, Sterculiaceae.

b. Flora: De acuerdo a lo descrito por Baca y Lozano (1999), se han determinado 74 familias botánicas, entre las que sobresalen, Lauraceae, Moraceae, Melastomataceae, Bromeliácea, Araceae y Orchidaceae, de las cuales hay identificadas 122 a nivel de 
género, distribuidas entre los estratos herbáceo, arbustivo, arbóreo y epifito.

c. Fauna: Según lo mencionado por Alegría y Ruiz (2002), la mayor parte de la fauna corresponde a especies generalistas y/o comunes, lo cual significa que ha ocurrido pérdida o desplazamiento de especies con requerimientos estrechos o especializados de nichos. La fauna está representada por mamíferos como ardillas (sciurus vulgaris), zarigüeyas (Didelphis marsupialis), conejos (Oryctolagus sp.) y ratones de campo (Apodemus sylvaticus), además de las trece especies de quirópteros pertenecientes a las familias Phyllostomidae, Vespertilionidae y Molossidae (Castro y Zura, 2000). Aves como barranqueros (Momotus aequatorialis), cerrajas (Cynocorax yncas), tancaras (Thraupidae sp.), azulejos (Thraupis episcopus), gorriones (Zonotrichia capensis), toriadores (Tyrannidae sp) y carpinteros (Colaptes $s p$ ). Insectos pertenecientes a los órdenes Lepidóptera, Orthoptera. Reptiles como falsa coral (Lampropeltis triangulum), jueteadora (Chironius montícola), coral (Micrurus sp), rabo de ají (Micrurus miparttitus), culebra huertera (Sibon nebulata) (Alegría y Ruiz, 2002). Méndez y Vallejo (2003) mencionan que la avifauna las familias más abundantes son Thraupidae, Fringillidae, Tyrannidae, Columbidae, Trochilidae, y Cuculidae. Siendo las familias relevantes para este estudio, las pertenecientes al Orden Apodiforme, la familia Trochilidae y Orden Passeriforme, las familias Traupidae, Fringilidae y Tyranidae. Por ejemplo, en elJBP, la especie de barranquero Momutus aequatorialis, perteneciente a la familia
Momotidae del Orden Coraciforme, es un ave simbólica y característica de la zona, la cual es muy llamativa por su tamaño y colores, además de la formación de raqueta en la punta de las plumas de la cola, la cual caracteriza a los individuos machos (dimorfismo sexual). Posee una peculiar forma de anidación, la cual se realiza en barrancos y despeñaderos.

Zonificación ecológica a partir de la caracterización de unidades de paisaje.

Recopilar y organizar los datos de campo permitió procesar y analizar la información geográfica que se muestra en el Mapa 2. Los resultados fueron: delimitación geográfica del área de estudio con un área de 44,4 ha. e identificación de una capa correspondiente a infraestructura del campus Los Robles de la FUP, con un área de 3,6 ha, distribuida en: oficinas administrativas, bienestar universitario, zonas recreativas, zonas verdes, zonas de parqueadero, laboratorios biología y química, cafetería, salones de clase y salas de sistemas.

Posteriormente se señaló la capa de vías, que contiene la zona para acceder a oficinas, salones, zonas de conservación y producción, etc. Finalmente, el mapa indica tres afluentes hídricos, recurso valioso para la zona de conservación: la quebrada Renacer hace parte del límite Noroccidente del campus, y es una fuente vital de la zona ya que atraviesa el JBP, con una longitud de $778 \mathrm{~m}$; quebrada Mano de Oso ubicada en el límite Sur, pasa por el bosque y tiene una longitud de $781 \mathrm{~m}$; quebrada Corazones, atraviesa el campus 


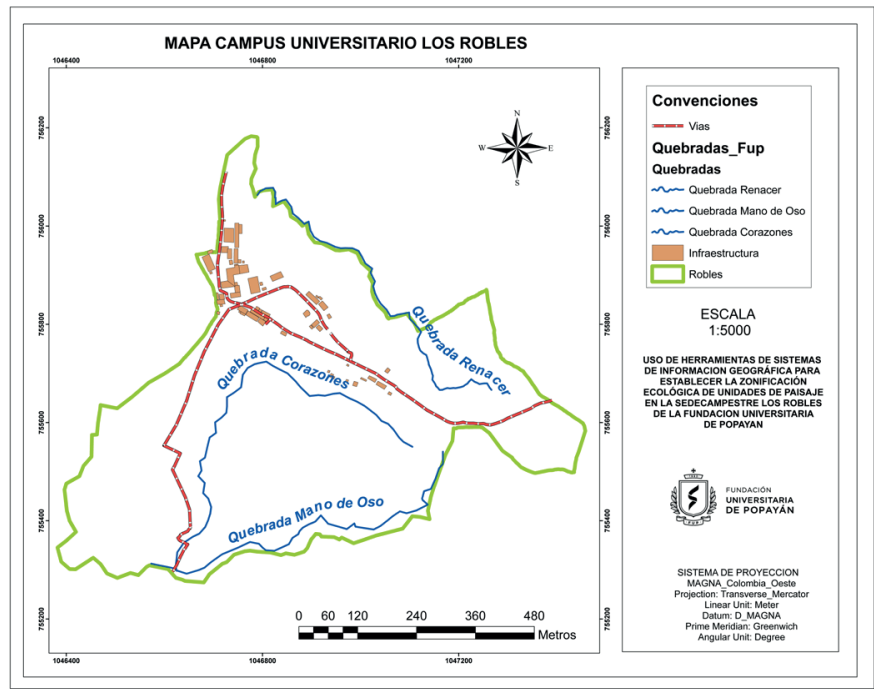

Mapa 2. Campus Universitario los Robles

universitario pasando por la zona de bosque, de paisaje con mayor extensión es la zona y limitando con áreas de potrero, tiene una longitud de $901 \mathrm{~m}$. de conservación con un área de 3,6 ha, esta extensión obedece a que en el área de estudio se encuentra elJardín Botánico En el Mapa 3 se presenta las principales de Popayán, por ende, los esfuerzos de unidades de paisaje que conforman el área conservación se ven evidenciados en el de estudio. Se puede apreciar que la unidad terreno real. De igual forma la vocación

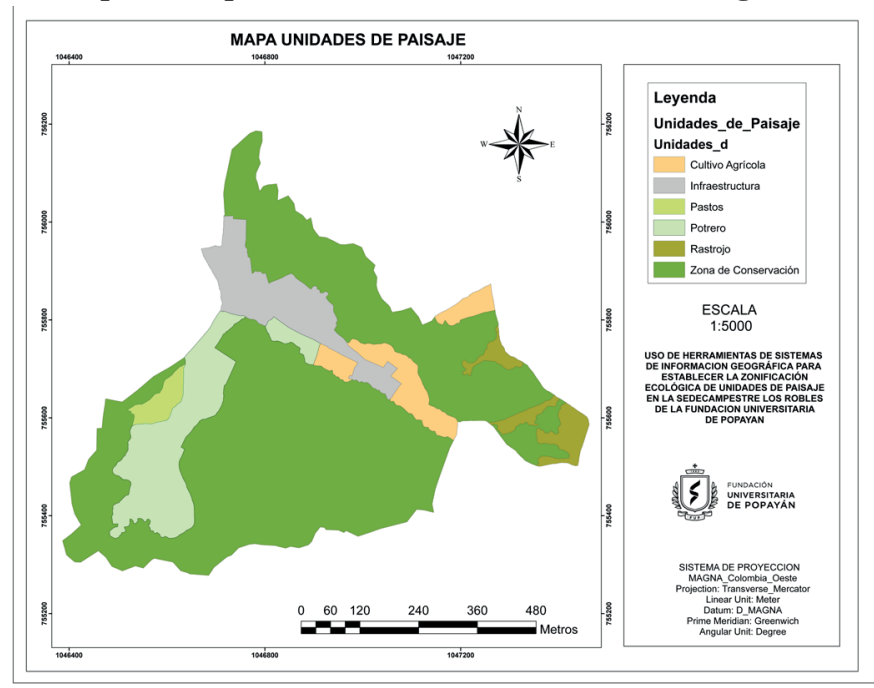

Mapa 3. Unidades de paisaje 
de uso de suelo corresponde a la agroforestería, por eso hay más bosque que cultivos agrícolas.

Las coberturas vegetales identificadas (Mapa 4), son el bosque fragmentado con pastos y cultivos con un área de 38,64 ha, que sobrepasa en extensión las demás coberturas, ya que comprende territorios naturales que han tenido intervención humana. De acuerdo a lo mencionado por Calambás, (2018), la Quebrada Mano de Oso es subcuenca de la quebrada Renacer y se encuentra ubicada al Sur del Jardín Botánico, su longitud dentro del campus es de 781 metros y cuenta con un parche de bosque denso, con buen estado de conservación y alta presencia de epifitas vasculares y no vasculares. De igual manera, alberga cerca de 44 especies de plantas leñosas, representadas principalmente por su abundancia de Palicourea thyrsiflora (cafetillo) y Meriania speciosa (flor de mayo) (Chaves \& Hurtado, 2019) y alta presencia de la planta Oreopanax floribundum (mano de oso) a la que debe su nombre.

Cabe mencionar que las zonas naturales corresponden al JBP y al bosque con vegetación secundaria, sin embargo, alrededor de estos se llevan a cabo diferentes actividades agropecuarias en las que se encuentran cultivos de café y plátano, cultivos que requieren manejo y fertilización, acciones que afectan el hábitat de las especies y la transformación de la estructura del bosque. El mosaico de cultivos tiene un área de 2,1 ha constituido por cultivos anuales, transitorios y permanentes, el tamaño de las parcelas es menor que 25 ha. Cabe mencionar que esta cobertura representa un área menor evidenciada en el mapa ya que los suelos son superficiales a profundos, bien drenados,

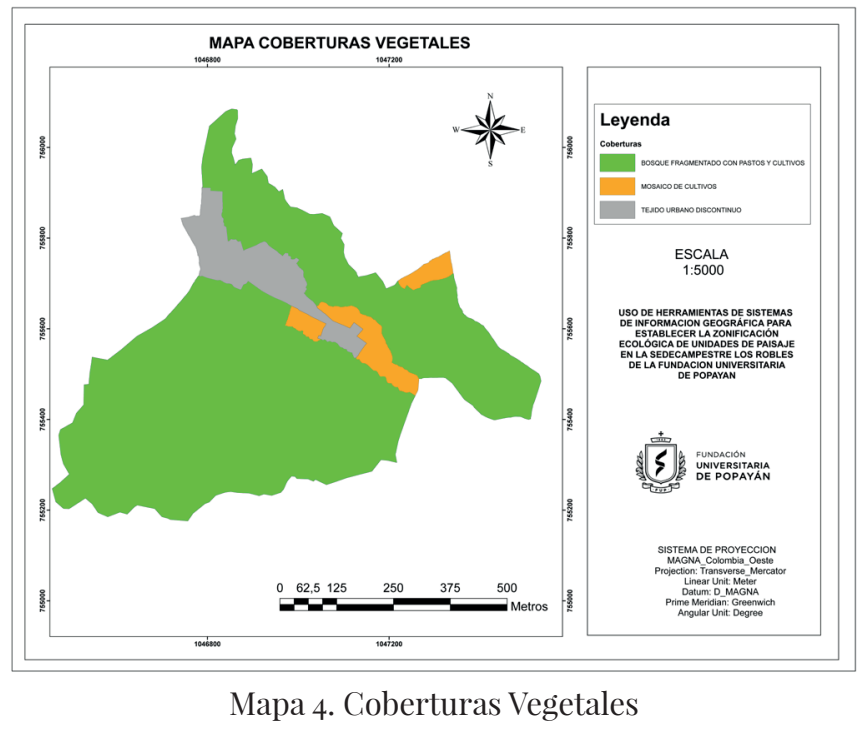


de textura moderadamente gruesa finas, asimismo, son tierras con fertilidad de baja a moderada, lo que no permite tener parcelas extensas de cultivos en el campus y por ende, una elevada producción.

En el Mapa 5 se muestra el uso actual del suelo, donde se identifican todas las parcelas que componen el paisaje del área de estudio. La extensión de los cultivos es menor, ya que los requerimientos de fertilidad son bajos. Sin embargo, los potreros y bosque con vegetación secundaria tienen mayores extensiones dada la aptitud de suelo y el origen inicial del bosque. Los pastos y rastrojos representan una extensión moderada, por la aptitud de suelo y sus bajos requerimientos de fertilidad y manejo.

El Mapa 6 corresponde a la aptitud del suelo. Aquí se observa la división del terreno en cuatro categorías, identificadas a partir de las características propias de cada unidad del paisaje definidas en el estudio general de suelos y zonificación de tierras para el departamento del Cauca a Escala 1:100.0oo de 2009. Se identificaron así: aptitud agrícola con una extensión de 2.5 ha, conformado por pequeños terrenos destinados a la producción a baja escala; aptitud para la conservación, 37,9 ha correspondiente a las zonas naturales y bosque secundario; aptitud para infraestructura con área de 3,6 ha, que corresponde a toda la planta física del campus Los Robles de la FUP; aptitud para rastrojo, o,3 aunque no tiene una finalidad específica, se proponen planes de restauración y corredores biológicos ya que limita con la zona de conservación y bosque secundario ya mencionada.

En cuanto al uso del suelo las tierras están utilizadas en ganadería extensiva y agricultura con cultivos de café, caña de azúcar, yuca, plátano, frutales y algunos lotes reforestados o con explotaciones forestales.

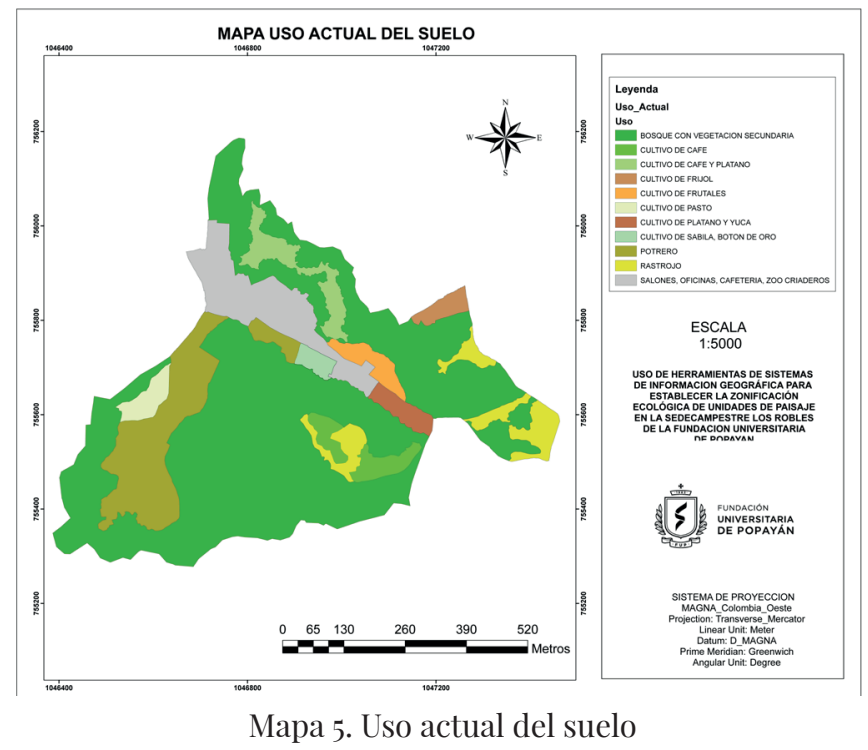




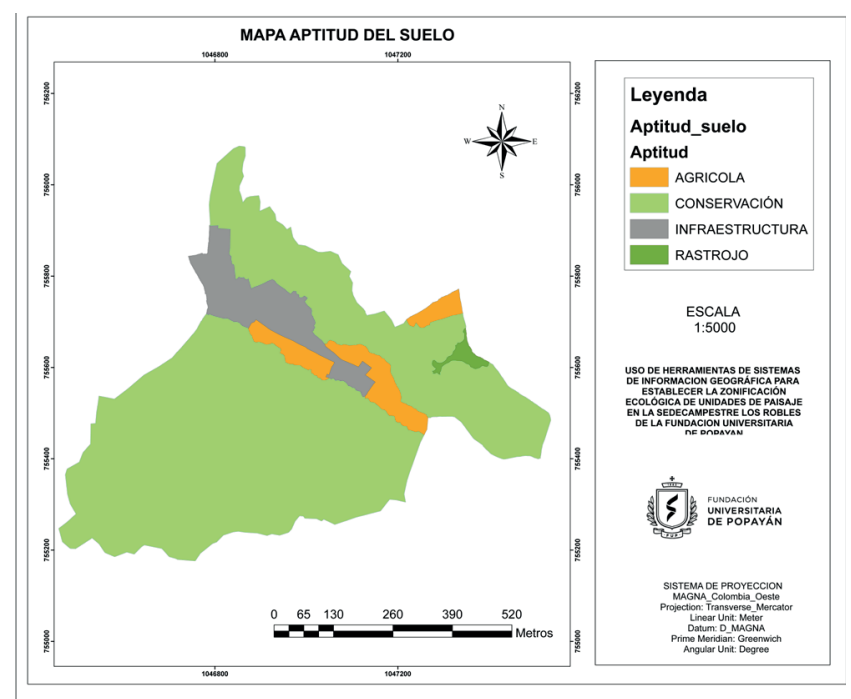

Mapa 6. Mapa aptitud del suelo

Estas tierras tienen aptitud para ganadería extensiva en pastos introducidos, para algunos cultivos densos, algunos sistemas agroforestales y plantaciones forestales. Requieren prácticas de conservación como rotación de cultivos, siembra en contorno o en fajas, aplicación de fertilizantes teniendo en cuenta la capacidad de fijación de fósforos, aplicación de cal, construcción de sequias de ladera y adecuado manejo de pastos y ganados (IGAG, 2009). Según lo mencionado por Albear et al., (2002), las tierras del municipio de Timbío tienen vocación de agroforestería por su característica biofísica (clima, relieve, material parental, suelos, erosión) lo cual no permite la utilización exclusiva de usos agrícolas o ganaderos. Estas tierras deben ser utilizadas bajos sistemas combinados, donde deliberadamente, se mezclen actividades agrícolas, ganaderas y forestales, en arreglos tanto especiales como temporales. Los sistemas agroforestales implican una interacción ecológica, productiva entre el componente forestal, agrícolas y/o pecuarios. Los objetivos de los sistemas agroforestales son preservar la biodiversidad, conservar o propiciar un microclima, aumentar la productividad vegetal o animal, aumentar la sostenibilidad de la explotación, diversificar la producción. Integrar la producción forestal con la producción pecuaria, disminuir los riesgos del agricultor, mitigar los efectos perjudiciales del sol, viento y las lluvias sobre el suelo.

En el mapa 7 se evidencia el conflicto de uso de suelo y se señalan los problemas más relevantes dentro del campus universitario. El mapa se obtuvo al intersecar el mapa de uso actual y el mapa de aptitud del suelo, 
paso importante para analizar la situación ambiental. Como resultado se identificaron los criterios de clasificación, a partir del análisis del territorio, metodologías de la FAO y del Plan Básico de Ordenamiento Territorial (PBOT) del municipio de Timbío para 2006 en su capítulo de "usos potenciales del suelo y síntesis de la aptitud de los suelos para la zonificación rural". En primera medida de define el uso adecuado, este corresponde a la óptima utilización del suelo en cuanto a manejo y vocación. Seguidamente el sobreuso o sobre carga a las condiciones del terreno, en este criterio se tiene en cuenta las acciones para mejorar el suelo. Finalmente, el uso inadecuado o inapropiado para la vocación y condiciones edáficas del suelo, en este criterio se debe cambiar las actividades y prácticas que se realizan en esa zona.
Cabe mencionar que el mapa de conflicto de uso, permitió tomar decisiones de forma integrada, así como la identificación de las unidades de área de estudio para su posterior zonificación ecológica. Se evidencia 37,7 Ha del uso adecuado del terreno, este corresponde a las zonas de conservación, infraestructuray zoo criaderos. El sobreuso con 7,6 ha corresponde a los cultivos de café, que no deberían estar en las zonas naturales, ya que su manejo y producción afectan el flujo natural de los ciclos de vida. Este criterio también se encuentra en áreas como potreros, la cual debe ser usada para establecer un corredor biológico delJBP para que conecte algunos fragmentos de bosque natural. Por último, el uso inadecuado, hace alusión a las zonas de rastrojo que deberían ser recuperadas y la zona de potrero que debería conservar la vegetación inicial.

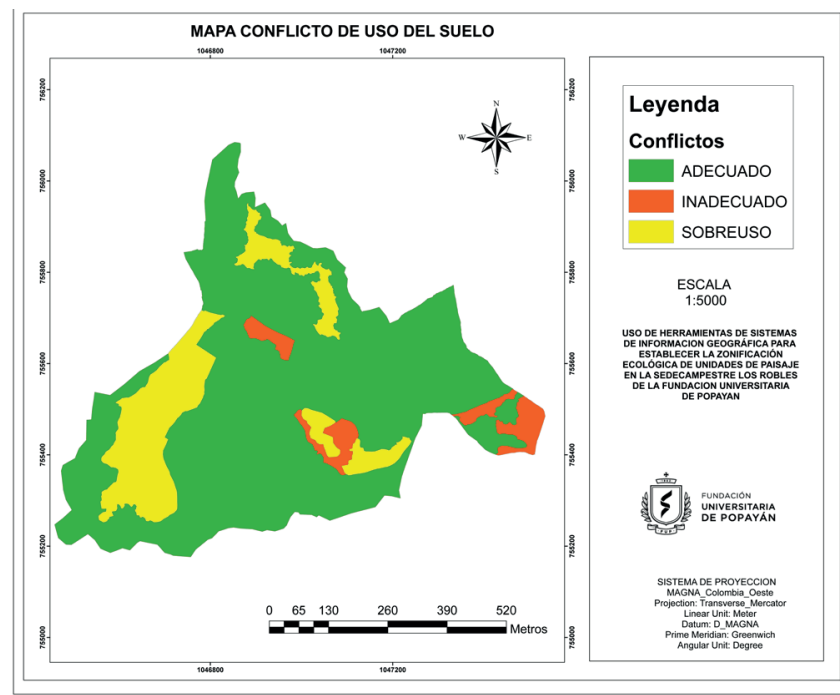

Mapa 7. Conflicto de uso del suelo. 
En el mapa 8 se muestra la zonificación ecológica, condiciones físicas y aptitud de suelos que tiene el terreno. Se evidencia que en el mapa no se propuso la conservación para toda el área de estudio, sino que se respetó la organización territorial inicial, que cumple diferentes funciones. Sin embargo, la zonificación se hace con el firme propósito de que se conserve el área de interés ambiental y de que no se amplíe la zona de producción económica para no genere más impactos ambientales negativos.

También se presenta la zona de restauración, ya que se identificaron cultivos de café en la zona del JBP, pues el manejo agrícola puede intervenir en algunas conexiones con el ecosistema acuático y terrestre. Se propuso el establecimiento de barreras vivas corta vientos para el área de potrero. Seguidamente en la zona de riesgo y amenaza se propone realizar una obra biomecánica ya que en la zona de estudio se originan deslizamiento en tiempo de alta precipitación que afectan el paso por el sendero ecológico delJBP. Cabe destacar que la zona de especial significado ambiental, tiene atributos ecológicos importantes para la funcionalidad del paisaje.

De acuerdo a lo expuesto por Baca y Lozano (1999) la vegetación del Jardín Botánico es muy biodiversa pues posee familias botánicas desde la Araceae, Asteraceae, Bromeliaceae, Lauraceae, Moraceae, Melastomataceae, Orquidaceae, Poaceae hasta la Rubiaceae. Los individuos de estas familias están distribuidos en los estratos epífito, trepador rasante, herbáceo, arbustivo y arbóreo. Según Cantor y Urbano (2002), en los diferentes estratos se observa vegetación asociada con orquídeas, bromelias, musgos, líquenes, helechos, bejucos y lianas. Además,

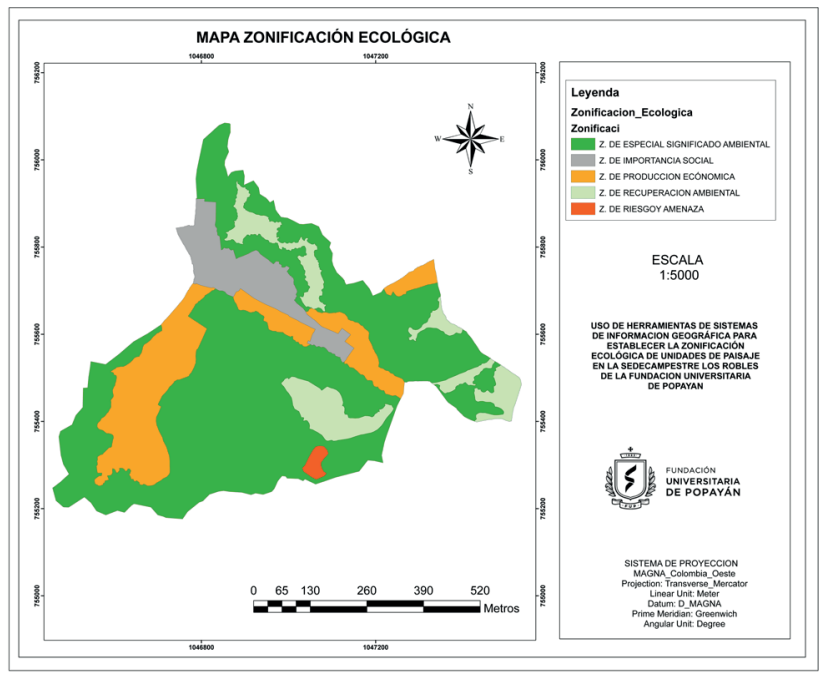

Mapa 8. Zonificación Ecológica 
el área de estudio tiene biodiversidad alta en líquenes que, de acuerdo al estudio realizado por Chilito et al., (2015) se encuentran 63 especies registradas de líquenes, de los cuales 39 están identificada hasta especie, 21 hasta género, tres no fueron determinadas y pertenecen a 29 géneros y 17 familias. Entre las familias se destacan: Ramalinaceae, Brigantiaeaceae, Parmeliaceae Coccocarpiaceae, Coenogoniaceae, Arthoniaceae, Lobariaceae, Graphidaceae, Haematommataceae, Arthoniaceae, Physciaceae, Parmeliaceae, Lecanoraceae, Collemataceae, Malmideaceae, Pannariaceae, Porinaceae, Teloschistaceae.

También es importante resaltar la biodiversidad de aves que habitan y visitan el JBP. Según Vargas, (2014) se registran la familia Traupidea (tangaras) con siete especies, la cual tiene la mayor abundancia con 21 individuos, seguido por la familia Turdidae (mirlas) con 14 individuos Trochilidae (colibríes) con 12 individuos y Tyrannidae (atrapamosca) con 10 individuos. Las tres familias restantes presentaron una abundancia menor. Las especies más representativas respeto a su abundancia, fueron Turdus ignobilis con 10 individuos, seguido por Zimmerius chrysops con ocho individuos y Thraupis episcopus con siete individuos.

Establecimiento de unidades de manejo que permitan alternativas de conservación.

Las unidades de manejo se delimitan a partir de la información contenida en el
Mapa 7 Conflicto de uso de suelo en el Mapa 7 y en el Mapa 8 de zonificación ecológica. Estas unidades van muy ligadas a acciones que determinan y evalúan el estado natural de las zonas. En las de uso inadecuado, se proponen planes de restauración ecológica con especies nativas que no afecten el desarrollo natural del bosque. En las zonas de sobreuso se propone recuperar el suelo con correcciones de acidez, fertilización orgánica y rotación de cultivos.

\section{Estrategias de conservación propuestas}

Por cada una de las zonas identificadas, se proponen las siguientes alternativas de conservación que apoyen el manejo a mediano y largo plazo de forma integral con el territorio:

\section{Zona de especial significado ambiental:}

1.Defender procesos y sistemas biológicos que sustenten la vida de los cuales depende el desarrollo del ser humano.

2.Preservar la diversidad genética necesarios para la protección de fauna y flora.

3.Iniciar y adaptar proyectos al desarrollo sostenible a mediana y gran escala.

4.Mantener la capacidad productiva de los ecosistemas, asegurando la disponibilidad de recursos: agua, flora y fauna.

5.Motivar la investigación ecológica plantaanimal. 
Zona de importancia social:

1.Lograr el reconocimiento del valor ecológico de las zonas naturales y de conservación por parte de los habitantes de la zona.

2.Hacer uso razonable de los bienes y servicios empleados para el desarrollo de actividades productivas.

3.Desarrollar estrategias para la amplia divulgación de las políticas ambientales de cada empresa o proyecto productivo.

Zonas de producción económica:

1.Realizar buenas prácticas ambientales en las parcelas de cultivo.

2.Proponer y cumplir acciones encaminadas a la fertilización y producción orgánica de productos.

3.No ampliar las zonas de producción agrícola, de tal forma que en los cultivos que ya se tienen se optimicen procesos y productos.

Zonas de recuperación ambiental:

1.Evitar acciones que provoquen contaminación de cualquier tipo en la zona.

2.Ejercer planes de restauración ecológica con especies nativas.

3.Monitorizar y hacer seguimiento continuo de especies con especial significado funcional
Zonas de riesgo y amenaza:

1.Identificar en la zona de estudio riesgos y amenazas para la comunidad humana y vida silvestre.

2.Establecer políticas locales para el uso sostenible de los recursos naturales.

Estrategias de conservación para el jardín botánico de Popayán.

La zonificación ecológica desarrollada en esta investigación (Mapa 8), presenta la realidad actual de la zona de estudio, elemento estratégico para tomar decisiones en temas de ordenamiento ambiental territorial. Del mismo modo, permite que se apoyen y respalden estrategias de conservación enmarcadas en las líneas operativas establecidas en el Plan Nacional de Jardines botánicos.

Los jardines botánicos cumplen un papel muy importante en la conservación de los recursos genéticos a través de sus progiramas de investigación, educación, entrenamiento, conservación ex-situ e in-situ, expediciones al campo, etc. Para muchas plantas el cultivo en un jardín botánico puede ser la única opción de supervivencia. De acuerdo a lo mencionado por Marín y Salazar, (2020) los jardines botánicos promueven investigación relacionada con la conservación en aspectos tales como producción de semillas, dispersión, mecanismos de polinización y fenología.

En la Tabla 1 se muestran las estrategias de conservación que se proponen, a partir 
Tabla 1. Estrategias de Conservación para fortalecimiento del Jardín Botánico

\begin{tabular}{|c|c|c|c|}
\hline $\begin{array}{l}\text { Línea de } \\
\text { operación }\end{array}$ & Proyecto & Objetivos & Actividades \\
\hline \multirow{3}{*}{$\begin{array}{l}\text { Línea de } \\
\text { investigación }\end{array}$} & \multirow{3}{*}{$\begin{array}{l}\text { La } \\
\text { biodiversidad } \\
\text { como } \\
\text { estrategia de } \\
\text { conservación } \\
\text { en elJBP }\end{array}$} & \multirow{3}{*}{$\begin{array}{c}\text { Hacer estudios } \\
\text { poblacionales } \\
\text { de flora y fauna } \\
\text { del JBP }\end{array}$} & $\begin{array}{l}\text { Fortalecer los semilleros } \\
\text { interdisciplinarios de investigación }\end{array}$ \\
\hline & & & $\begin{array}{c}\text { Identificar especies claves en los } \\
\text { diferentes ecosistemas. }\end{array}$ \\
\hline & & & $\begin{array}{l}\text { Realizar convenios con universidades } \\
\text { locales y regionales para que sus } \\
\text { estudiantes realicen investigaciones y } \\
\text { trabajo de grado. }\end{array}$ \\
\hline \multirow{7}{*}{$\begin{array}{l}\text { Línea de } \\
\text { educación } \\
\text { ambiental }\end{array}$} & \multirow{7}{*}{$\begin{array}{l}\text { La educación } \\
\text { ambiental } \\
\text { como } \\
\text { herramienta } \\
\text { pedagógica } \\
\text { para la } \\
\text { conservación } \\
\text { de la } \\
\text { biodiversidad. }\end{array}$} & \multirow{7}{*}{$\begin{array}{l}\text { Diseñar } \\
\text { programas } \\
\text { de formación } \\
\text { ambiental } \\
\text { en contextos } \\
\text { escolares y } \\
\text { universitarios. }\end{array}$} & $\begin{array}{c}\text { Capacitar a estudiantes y docentes en } \\
\text { componentes de investigación en líneas } \\
\text { de biodiversidad, conservación de flora } \\
\text { y fauna. }\end{array}$ \\
\hline & & & $\begin{array}{l}\text { Formulación de estrategias para la } \\
\text { recuperación y manejo de especies a } \\
\text { través de convenios interinstitucionales. }\end{array}$ \\
\hline & & & $\begin{array}{c}\text { Publicar y dar a conocer las } \\
\text { investigaciones, en revistas, congresos, } \\
\text { seminarios. }\end{array}$ \\
\hline & & & $\begin{array}{l}\text { Realizar a nivel interno talleres y } \\
\text { capacitaciones para que el personal del } \\
\text { JBP conozca y se apropie de los procesos } \\
\text { que se desarrollan, servicios que se } \\
\text { ofrecen, con el objetivo de difundir este } \\
\text { conocimiento a propios y visitantes. }\end{array}$ \\
\hline & & & $\begin{array}{l}\text { Actualizar constante ente las colecciones } \\
\text { ex situ e in situ del JBP }\end{array}$ \\
\hline & & & $\begin{array}{c}\text { Implementar una estrategia } \\
\text { comunicacional para dar a conocer el } \\
\text { JBP en medios de comunicación locales y } \\
\text { redes sociales. }\end{array}$ \\
\hline & & & $\begin{array}{l}\text { Como resultado de las investigaciones, } \\
\text { publicar guías rápidas de flora y fauna. }\end{array}$ \\
\hline
\end{tabular}




\begin{tabular}{|c|c|c|c|}
\hline \multirow{5}{*}{$\begin{array}{l}\text { Línea de uso } \\
\text { sostenible } \\
\text { de la } \\
\text { biodiversidad }\end{array}$} & \multirow{5}{*}{$\begin{array}{c}\text { El desarrollo } \\
\text { sostenible } \\
\text { un tema } \\
\text { importante } \\
\text { en la } \\
\text { conservación } \\
\text { de la } \\
\text { biodiversidad. }\end{array}$} & \multirow{5}{*}{$\begin{array}{c}\text { Reconocer la } \\
\text { importancia y } \\
\text { funcionalidad } \\
\text { del desarrollo } \\
\text { sostenible, } \\
\text { como } \\
\text { estrategia } \\
\text { activa de } \\
\text { conservación. }\end{array}$} & $\begin{array}{l}\text { Identificar los objetos de fauna y flora y } \\
\text { su estado de vulnerabilidad en el JBP. }\end{array}$ \\
\hline & & & $\begin{array}{l}\text { Dar a conocer la importancia de las zonas } \\
\text { de especial interés ambiental a toda la } \\
\text { comunidad en general, para establecer } \\
\text { estrategias alcanzables de conservación. }\end{array}$ \\
\hline & & & $\begin{array}{c}\text { Restaurar zonas de recuperación } \\
\text { ambiental con especies endémicas de la } \\
\text { zona, con el objetivo de atraer especies } \\
\text { asociadas. }\end{array}$ \\
\hline & & & $\begin{array}{c}\text { Potenciar el Jardín Botánico de Popayán, } \\
\text { como lugar de avistamiento de aves, dado } \\
\text { que se registra cantidad y diversidad de } \\
\text { estas. }\end{array}$ \\
\hline & & & $\begin{array}{l}\text { Formular proyectos con fines de } \\
\text { protección - producción, con el objetivo } \\
\text { que generar ingresos que permitan } \\
\text { financiar los procesos de conservación. }\end{array}$ \\
\hline
\end{tabular}

del Plan Nacional de Jardines Botánicos (Conocimiento, conservación, valoración y aprovechamiento de la diversidad vegetal). Por lo tanto, se propuso trabajar tres de líneas operativas. 1. Línea de investigación, 2. Línea de educación ambiental. 3. Línea de uso sostenible de la biodiversidad. Cabe destacar que son líneas que obedecen a las necesidades y condiciones que la zona de estudio ofrece. Para lograr el objetivo se proponen proyectos a corto, mediano y largo plazo.

\section{Conclusiones}

El uso de vehículos aéreos no tripulados (drones) en estudios de zonificación ecológica permite la obtención de productos fotogramétricos de alta resolución espacial, disminuyendo tiempos y costos en levantamientos topográficos, obteniendo productos geoespaciales valiosos para realizar la fotointerpretación y generar cartografía digital que permitirá apoyar procesos en la toma de decisiones y ordenación ambiental de territorio.

La zonificación ecológica y el análisis del terreno, permitieron establecer áreas de especial significado ambiental; importancia social; recuperación ambiental; riesgo y amenaza; y producción económica en el campus universitario Los Robles de la FUP. Esto evidenció que la zonificación propicia la comprensión integral de los atributos que conforman la realidad del paisaje, con el fin de no eliminar ningún área, si no de darle un manejo adecuado que no irrumpa con el desarrollo de cada una. 
La intervención antrópica en zonas de interés ambiental trae consecuencias en la transformación del paisaje. Inicialmente se modifica la vocación del suelo, hecho que altera el flujo y desarrollo natural de las especies que lo habitan. Esto da como resultado cambios en la fisionomía, estructura y composición de las coberturas vegetales.

Resultado de esta investigación, se concluye que el campus universitario Los Robles, requiere de un instrumento de planificación que permita el logro de los objetivos de conservación y la construcción constante de estrategias que promuevan la conexión ecológica entre ecosistemas, en elJardín Botánico de Popayán y los bosques presentes en la zona.

La generación de estrategias de conservación para el JBP es importante ya que es una zona legalmente constituida dentro del Plan Nacional de Colecciones para Los Jardines Botánicos de Colombia. Así mismo, según lo mencionan Vargas et al. (2020) es una zona de especial interés ambiental pues alberga ecosistemas estratégicos; rondas; corredores biológicos; zonas con especies endémicas, amenazadas o en peligro crítico; áreas de importancia para cría, reproducción, alimentación y anidación; zonas de paso de especies migratorias.

\section{Agradecimientos}

Al programa Innovacción Cauca - Gobernación del Cauca y al programa de Ecología de la Fundación Universitaria de Popayán por haber brindado la posibilidad de realizar este proyecto de Investigación y a Alexander Medina por su acompañamiento y apoyo en las salidas de campo.

\section{Bibliografía}

Albear, D. Anchico, G. Cruz, M. Mosquera, C. Zapata, F. González, O. 2002. Zonificación y análisis ecológico de la zona Timbío - sur de Popayán con la utilización de sensores remotos, SIGy una perspectiva desde la ecología del paisaje. Trabajo de Grado en Ecología. Fundación 
Universitaria de Popayán, Facultad de Ciencias Naturales, Programa de Ecología. Popayán - Cauca. 124 pp.

Agredo,J. 2006. Zonificación ecológica como herramienta para establecer los lineamientos básicos para la formulación del plan de manejo del ecosistema de paramo, de la cuenca del rio piedras municipio de Popayán. Trabajo de Grado en Ecología. Fundación Universitaria de Popayán, Facultad de Ciencias Naturales, Programa de Ecología. Popayán - Cauca. 102 pp.

Alegría, B., Ruiz, Z. 2002. Composición y estructura de la avifauna en cafetales con sombrío, Vereda el Hato, Municipio de Timbío, Cauca, Colombia. Trabajo de Grado en Ecología. Fundación Universitaria de Popayán, Facultad de Ciencias Naturales, Programa de Ecología. Popayán - Cauca. 116 pp.

Baca, A. y Lozano, L.1999. Estructura y composición florística del área del Jardín Botánico de Popayán. Pp:25-30. Memorias, I CONGRESO COLOMBIANO DE BOTÁNICA. Santa de Bogotá: Universidad Nacional de Colombia. 85pp

Bambagüé, C y Arboleda, E. 2017. Zonificación y análisis ecológico de las unidades de paisaje mediante la aplicación de los sistemas de información geográfico. Trabajo de Grado en Ecología. Fundación Universitaria de Popayán, Facultad de Ciencias Naturales, Programa de Ecología. Popayán - Cauca. $102 \mathrm{pp}$.

Calambás, H. 2018. Evaluación del estado eco-sistémico de las coberturas vegetales asociadas a la Quebrada Renacer del Jardín Botánico de Popayán mediante bioindicadores ecológicos de la Familia (Formicidae: Hymenoptera). Tesis de pregrado Ecología. Fundación Universitaria de Popayán. Popayán - Cauca. 109 pp.

Cantor, S. y Urbano, S. 2002. Estudio de líquenes como bioindicadores de calidad de aire en la ciudad de Popayán, Departamento del Cauca, Colombia. Trabajo de Grado en Ecología. Fundación Universitaria de Popayán, Facultad de Ciencias Naturales, Programa de Ecología. Popayán - Cauca. 102 pp. 
Colombia. Ministerio de Ambiente, Vivienda y Desarrollo Territorial [Internet]. 2010. Metodología general para la presentación de estudios ambientales. Bogotá, D.C.: Colombia. Ministerio de Ambiente, Vivienda y Desarrollo Territorial, $72 \mathrm{p}$. Disponible en: http://portal.anla.gov.co/documentos/normativa/ metodologia_presentacion_ea.pdf

Cuatrecasas, J. 1958. Aspectos de la vegetación natural de Colombia; separata de las revistas de la Academia Colombiana de Ciencias exactas, físicas y naturales. Vol X, No 40. Edit. Voluntad; Bogotá. 21p.

Chilito, L., Soto, E, Peña, A. 2016. Efectos del microclima en la diversidad de especies y rasgos funcionales de líquenes cortícolas en el jardín botánico de Popayán (Cauca, Colombia), Cryptogamie, Mycologie, 37 (2), 205-215. Cryptogamie, mycologie 37 (2), 205-215, https://doi.org/10.7872/crym/v37.iss2.2016.205

Chaves, S. y Hurtado L. 2019. Estructura y composición de tres zonas boscosas del Fardín Botánico del campus Los Robles, Fundación Universitaria de Popayán. Trabajo de Grado en, Universidad del Cauca, Facultad de Ciencias Agrarias, Programa de Ingeniería Forestal. Popayán - Cauca. 102 pp.

Güetio, F y Solarte, W. 200o. Densidad florística en el área de influencia Wettu, como elemento para identificación de bancos de germoplasma "in situ" en el Jardín Botánico de Popayán. Trabajo de Grado en Ecología. Fundación Universitaria de Popayán, Facultad de Ciencias Naturales, Programa de Ecología. Popayán - Cauca. 102 pp.

IGAC. Instituto Geográfico Agustín Codazzi. 2009. Estudio general de suelos y zonificación de tierras. Departamento del Cauca. Impresa Nacional de Colombia, Bogotá. Colombia.

IGAC. Instituto Geográfico Agustín Codazzi. 2007. Fundamentos Físicos de Teledetección. Bogotá D.C: Telecentro Regional en Tecnologías Geoespaciales. p 13-15. 
Juspian, Y. 2019. Diversidad y ecología de los macrohongos (Basidiomycota y Ascomycota) en el bosque subandino del jardín Botánico de Popayán de la Fundación Universitaria de Popayán, Sede Los Robles, Timbío - Cauca. Tesis de pregrado Ecología. Fundación Universitaria de Popayán, Popayán, Colombia. 180 pp.

López, A. 2008. Aproximaciones conceptuales y metodológicas en la identificación de requerimientos para la conceptualización de un sistema de información geográfica participativo en el resguardo indígena Ticuna Uitoto kilómetros 6 y 11 carretera Leticia - Tarapaca. Trabajo de Grado Maestría. Universidad Distrital FranciscoJosé de Caldas. Facultad de Ingeniería

Programa de Ingeniería Catastral y geodesia. BogotáCundinamarca.128pp

Marín, M. y Salazar, A. 2020. Lineamientos generales para la planeación estratégica del Jardín Botánico de Popayán, sede Los Robles de la Fundación Universitaria de Popayán, Municipio de Timbío - Cauca. Tesis de pregrado Ecología. Fundación Universitaria de Popayán, Popayán, Colombia.

Méndez, P., y Vallejo, M. 2003. Evaluación de la presencia de líquenes foliosos cortícolas en las especies Pinus oocarpo y Herocarpus Americanus en el Jardín Botánico de Popayán. Trabajo de Grado en Ecología. Fundación Universitaria de Popayán, Facultad de Ciencias Naturales, Programa de Ecología. Popayán - Cauca. 102 pp.

Negret y Torres. 1997. Caracterización climática del altiplano de Popayán. Museo de historia natural, Universidad del Cauca, CRC.

Ortiz, P. 2014. Estado de conservación del paisaje de la hacienda los Robles, vereda los Robles, Municipio de Timbío, Departamento del cauca. Trabajo de Grado en Ecología. Fundación Universitaria de Popayán, Facultad de Ciencias Naturales, Programa de Ecología. Popayán - Cauca. 102 pp. 
Planes de Ordenamiento y Manejo de Cuencas Hidrográficas POMCA [Internet]. 2007. Planes de ordenamiento y manejo de cuencas hidrográficas de los ríos Sambingo-Hato viejo, municipios de Bolívar, Mercaderes y Florencia, departamento del Cauca. Disponible en: http://web2018.crc.gov.co/index.php/ ambiental/planes-de-ordenacion-y-manejo-de-cuencashidrograficas/167-rio-sambingo-hato-viejo

Samper, J. 2015. Plan nacional para el fortalecimiento de las colecciones vivas en los Jardines Botánicos de Colombia. Tesis maestría Gestión Ambiental, Pontificia universidad Javeriana, Facultad de Estudios Ambientales y Rurales. Bogotá - Cundinamarca. 125pp.

Serrato, P. 2018. Zonificación ecológica como base para el diagnóstico de cuencas hidrográficas Proyecto: Estudio de imágenes satelitales en zonas tropicales para estudios geomorfológicos y de cobertura. Instituto Geográfico Agustín Codazzi. Oficina CIAF. Bogotá.31pp.

Vargas,J. 2014. Estudio y comparación de la comunidad de aves (Passeriforme y Apidiforme) perteneciente al piso climático sub-Andino en la Fundación universitaria de Popayán sede Los Robles Popayán, Cauca. Trabajo de Grado en Ecología. Fundación Universitaria de Popayán, Facultad de Ciencias Naturales, Programa de Ecología. Popayán - Cauca. 111 pp.

Vargas, J., Feriz, D., Chilito, L. y Chacón, J. 2020. Diversidad de aves del Jardín Botánico de Popayán, en la Fundación Universitaria de Popayán. Rev. ConCiencia. ISSN 2256-1625. Vol.10.Junio de 2020, págs. 8 - 27.

Villegas, E, Gifuentes, A, Contreras, G, y Fernández D. 2015. Ordenamiento territorial como instrumento para la zonificación ambiental a través de la Estructura Ecológica Principal. Universidad El Bosque Facultad de Ingeniería, Programa Ingeniería Ambiental, Revista de Tecnología, Journal of Technology, 14(2): 49-76. 